CYTA - Journal of Food

\title{
CONTENIDO DE MERCURIO EN CONSERVAS DE MEJILLONES, BERBERECHOS Y NAVAJAS COMERCIALIZADOS EN GALICIA (ESPAÑA) MERCURY CONTENT IN TINNED MUSSELS, COMMOM COCKLES AND RAZOR SHELLS COMMERCIALIZED IN GALICIA (SPAIN)
}

M. A. Fernández García , J. Alonso Díaz , L. Loira Torres \& M. J. Melgar Riol

To cite this article: M. A. Fernández García , J. Alonso Díaz, L. Loira Torres \& M. J. Melgar Riol (2007) CONTENIDO DE MERCURIO EN CONSERVAS DE MEJILLONES, BERBERECHOS Y NAVAJAS COMERCIALIZADOS EN GALICIA (ESPAÑA) MERCURY CONTENT IN TINNED MUSSELS, COMMOM COCKLES AND RAZOR SHELLS COMMERCIALIZED IN GALICIA (SPAIN), CYTA - Journal of Food, 5:5, 379-383, DOI: 10.1080/11358120709487716

To link to this article: https://doi.org/10.1080/11358120709487716

C. Copyright Taylor and Francis Group, LLC Published online: 02 Oct 2009.

Submit your article to this journal ए 


\title{
CONTENIDO DE MERCURIO EN CONSERVAS DE MEJILLONES, BERBERECHOS Y NAVAJAS COMERCIALIZADOS EN GALICIA(ESPAÑA)
}

\section{MERCURY CONTENT INTINNED MUSSELS, COMMOM COCKLES AND RAZOR SHELLS COMMERCIALIZED INGALICIA(SPAIN)}

\author{
García Fernández, M. A.; Alonso Díaz, J.; Loira Torres, L.; Melgar Riol, M. J.* \\ Área de Toxicología. Facultad de Veterinaria. Universidad de Santiago de Compostela (Campus de Lugo). C/ \\ Carvalho Calero s/n. 27002-LUGO, España.
}

Recibido/Received 21-03-2007; aceptado/accepted 18-06-2007

*Autor para la correspondencia/Corresponding author. E-mail: mjmelgar@lugo.usc.es

\begin{abstract}
Molluscs accumulate heavy metals and impose health hazard to consumers. Mercury is considered as a metal not essential and very toxic. Concentrations of mercury were determined for tinned mussels, common cockle and razors shell, commercialized in Galicia (Spain). Previous sample digestion process in acid medium in microwaves, for the analysis, an anodic stripping voltammetric technique, using a gold disc as working electrode, has been used in order to obtain the metal concentrations in muscle, liver and covering liquid. Results for complete molluscs, expressed in ppm and fresh weight, presented the greater levels in mussels (0.27), followed by common cockle $(0.20)$ and finally the razors shell $(0.08)$. In the portions, mercury contents were: liver $>$ covering liquid $>$ muscle. All observed concentrations of mercury were below the maximum limit permitted for human consumption; therefore, it does not constitute a risk for consumer health.
\end{abstract}

\section{Resumen}

Los moluscos acumulan metales pesados y ponen en riesgo la salud del consumidor. Entre estos metales, el mercurio está considerado como no esencial y muy tóxico. Se determinó la concentración de mercurio en mejillones, berberechos y navajas, en conservas comercializadas en Galicia (España). Las porciones de músculo, hepatopáncreas y líquido de cobertura se sometieron a un proceso de digestión, en medio ácido, en microondas. El mercurio se analizó por voltamperometría de redisolución anódica con electrodo de oro. Los resultados para molusco completo, expresados en ppm y peso fresco, mostraron los mayores niveles para mejillones $(0,27)$, seguidos de berberechos $(0,20)$ y finalmente las navajas $(0,08)$. El contenido de mercurio por porciones fue: hepatopáncreas $>$ líquido de cobertura $>$ músculo. Todas las concentraciones de mercurio estuvieron por debajo del límite máximo permitido para consumo humano. Por tanto, su consumo no supone riesgo alguno para la salud del consumidor.

Keywords: Metal, mercury, mussel, common cockle, razor shell Palabras clave: Metal, mercurio, mejillones, berberechos, navajas

\section{INTRODUCCIÓN}

La actividad humana ha contribuido decisivamente al aumento de las concentraciones de metales pesados en todos los ecosistemas hídricos. Esta contaminación ambiental es muy importante por su persistencia y por su posible vehiculación a los alimentos que ocasionaría perjuicio en la salud del consumidor. Algunos metales, entre ellos el mercurio, al ser ingeridos no tienen funciones metabólicas conocidas y son tóxicos a bajas dosis (Mas y Azcue, 1993). El mercurio es conocido, fundamentalmente como neurotóxico y teratogénico (metilmercurio), pero además afecta al tracto gastrointestinal y a la función renal
$\left(\mathrm{Hg}^{2+}\right)$ (Klaassen, 2001), por ello la EFSA (Autoridad Europea de Seguridad Alimentaria) aprobó una ingesta semanal tolerable provisional (PTWI) para el metilmercurio de 1,6 $\mu \mathrm{g} / \mathrm{kg}$ de peso corporal (PC) (DOUE, 2006). Por otra parte, las rías gallegas, como lugares de intercambio de aguas entre los estuarios de los ríos y el océano, tienen un interés especial por ser zonas habitualmente usadas para el cultivo de los moluscos destinados al consumo humano, entre éstos se encuentran: mejillones, berberechos y navajas (Prego, 1995).

El mecanismo de alimentación de los moluscos bivalvos requiere filtrar grandes cantidades de agua, lo que favorece la bioconcentración de contaminantes en 
cantidades superiores a las del medio donde viven, originando problemas de tipo sanitario (Vázquez, 2002).

La contaminación de los moluscos está en función, independientemente de su estado fisiológico, de la calidad y grado de contaminación que tenga el agua de mar en la que viven, afectándoles en mayor o menor grado (Gobert et al. 1992). Los moluscos se asientan y encuentran su hábitat natural en las zonas marítimas próximas a las costas porque son aguas ricas en plancton, con unas salinidades comprendidas entre $25-35 \%$, temperatura entre 11 y $21^{\circ} \mathrm{C}$ y el $\mathrm{O}_{2}$ disuelto entre 6 y $8,4 \mathrm{mg} / \mathrm{l}$; valores entre los que están comprendidos los óptimos de casi todos los moluscos. En estas aguas es donde se acumula en mayor grado la contaminación, por lo que los moluscos pueden ser víctimas de ella (Puerta, 1995).

Los moluscos son conocidos por tolerar y acumular altas concentraciones de contaminantes metálicos y orgánicos en la glándula digestiva (Domouhtsidou y Dimitriadis, 2000), ya que usan como mecanismo de regulación el almacenamiento de los tóxicos en el hepatopáncreas. Esta bioacumulación depende de factores bióticos, fundamentalmente la especie y otros como la edad, actividad reproductiva y talla del molusco. Así mismo, influyen factores abióticos tales como la época del año, salinidad, temperatura, presencia de otros metales en el agua y también la especiación química de los diferentes metales (Riisgård et al., 1985; Wang, 2001; Klaassen, 2001).

La producción de moluscos respecto a los compuestos tóxicos está sujeta a las normas de calidad de las aguas (BOE, 1993); pero además, existen normas aplicables a los límites de metales pesados en productos alimenticios, así, el Reglamento (CE) No 1881/2006 de la Comisión (DOUE, 2006) fija el contenido máximo de mercurio para productos de la pesca y carne de pescado en $0,50 \mathrm{mg} / \mathrm{kg}$ de peso fresco.

Algunos trabajos realizados en moluscos en conserva han mostrado que la cantidad acumulada en metales pesados, no reviste toxicidad para el consumidor al no sobrepasar los niveles establecidos (Gutiérrez et al., 2004, 2006; Hernández-Moreno et al., 2004, 2005; Hernández et al., 2006).

El objetivo de este estudio fue determinar los niveles de mercurio en muestras de mejillones, berberechos y navajas en conserva comercializadas en Galicia (España), para evaluar el riesgo toxicológico derivado de su consumo.

\section{MATERIALYMÉTODOS}

\section{Recogida de muestras}

El presente estudio se realizó utilizando mejillones, berberechos y navajas en conserva comercializados en Galicia. Los mejillones en 12 preparaciones culinarias: escabeche (5), al natural (1), escabeche y oliva (2), salsa a la gallega (1) y salsa de vieira (3), y los berberechos (3) y navajas (3) todas ellas al natural. Las muestras fueron elegidas mediante un proceso de aleatorización con SPSS. Se adquirieron en el año 2006 un total de 54 muestras (latas) en diferentes establecimientos de alimentación, para que este muestreo fuese lo más representativo posible cara al consumidor final.

\section{Técnica analítica}

Para cada una de las tres especies estudiadas se tomaron 3 latas idénticas de la misma marca y preparación (36 de mejillones, 9 de berberechos, y 9 de navajas). Se tomó cada molusco mediante una pinza adecuada y se lavó con agua ultrapura milli-Q para eliminar los restos de líquido de cobertura y de sólidos (cebolla, tomate, etc.). Se procedió a su disección separando la porción muscular y el hepatopáncreas (las navajas estaban enlatadas sin hepatopáncreas). Se juntaron las porciones similares de cada molusco, obteniendo un total de 5 muestras de cada marca, de las cuales dos muestras se corresponden con músculo, dos con hepatopáncreas y una con el líquido de cobertura (BOE, 2003). Todo el proceso se realizó por triplicado.

Las muestras fueron homogeneizadas en un homogeneizador de vidrio borosilicatado con émbolopistón en PTFE estriado, y posteriormente, digeridas en medio ácido (nítrico-clorhídrico) en una estación de microondas de laboratorio (ETHOS 20. Milestone), equipado con vasos cerrados de teflón TFM.

Una vez digeridas, las muestras fueron llevadas a unos matraces aforados y enrasadas con agua milli-Q a 50 $\mathrm{ml}$, quedando listas para el análisis por voltamperometría de redisolución anódica con electrodo rotatorio de oro. El barrido de potenciales aplicado osciló entre 500 y $700 \mathrm{mV}$ y las condiciones de trabajo adaptadas de las descritas por Alonso et al. (2000) y García-Vázquez (2005) fueron: desaireación: $180 \mathrm{~s}$, agitación: $120 \mathrm{~s}$, potencial de preconcentración: $370 \mathrm{mV}$, tiempo de preconcentración: 120 s, volumen de muestra: $1 \mathrm{ml}$. El potencial de pico para el mercurio se localizó a $600 \mathrm{mV}$.

Para el control de calidad del método fue empleado como material de referencia el liquen Evernia prunatri $L$. (IAEA-336) y se siguió la normativa vigente (DOCE, 1990) valorando los parámetros mostrados en la Tabla 1 mediante 12 réplicas de una misma disolución.

\section{Análisis estadístico}

Para el análisis estadístico de los resultados se utilizó el programa SPSS 12.0 para Windows, realizando el Test t para muestras independientes y ANOVA de un factor.

El ANOVA de un factor genera un análisis de la varianza para una variable dependiente cuantitativa (concentración de mercurio) respecto a una única variable de factor, la variable independiente (especie, porción o preparación).

El Test $t$ para muestras independientes se utilizó para verificar entre qué porciones hay diferencias significativas dentro de cada especie. 
Tabla 1. Resultados obtenidos en el control de calidad del método.

Table 1. Results obtained for quality control of the method.

\begin{tabular}{lc}
\hline Parámetro & Valor \\
\hline $\begin{array}{l}\text { Concentración del material } \\
\text { referencia certificado }(\mathrm{mg} / \mathrm{kg})\end{array}$ & 0,20 \\
Intervalo de confianza & $0,16-0,24$ \\
Concentración encontrada & $0,22 \pm 0,05$ \\
Recuperación $(\%)$ & $110 \%$ \\
\hline
\end{tabular}

\section{RESULTADOS Y DISCUSIÓN}

En general, cabe destacar en estas especies de moluscos, que las concentraciones en hepatopáncreas fueron más elevadas que en músculo y en líquido de cobertura. En mejillones, las concentraciones fueron: hepatopáncreas $>$ líquido de cobertura $>$ músculo; en berberechos: hepatopáncreas $>>$ líquido de cobertura > músculo y en navajas presentaron niveles muy similares tanto en músculo como en el líquido de cobertura.

Considerando las tres especies estudiadas y referido al molusco total o completo, teniendo en cuenta la participación relativa de las dos porciones $(80 \%$ músculo y $20 \%$ hepatopáncreas), los mejillones alcanzaron los niveles más altos $(0,27 \mathrm{mg} / \mathrm{kg}$ p.f.) seguido de berberechos $(0,20 \mathrm{mg} / \mathrm{kg}$ p.f. $)$ y finalmente las navajas $(0,08 \mathrm{mg} / \mathrm{kg}$ p.f. $)$ (Figura 1).

En cuanto a las formas de preparación, para el mejillón, la preparación «al natural» mostró el contenido más bajo al considerar el molusco completo-total, pero también en músculo y líquido de cobertura tomados por separado (Figura 2), este contenido inferior puede deberse a la simplicidad de la matriz.

Teniendo en cuenta la legislación europea vigente (DOUE, 2006) que establece como contenido máximo para productos de la pesca (entre los que están incluidos los moluscos en conserva) $0,5 \mathrm{mg} / \mathrm{kg}$ de peso fresco, solamente una muestra de mejillones en escabeche presenta una concentración más elevada, $0,64 \mathrm{mg} / \mathrm{kg}$ p.f., considerando el molusco total-completo, es decir, sumando la concentración encontrada en músculo $(0,46 \mathrm{mg} / \mathrm{kg}$ p.f.) y hepatopáncreas $(1,36 \mathrm{mg} / \mathrm{kg}$ p.f.) teniendo en cuenta el porcentaje que cada porción representa.

A los resultados obtenidos, respecto al contenido en mercurio, se les aplicó el tratamiento estadístico ANOVA de un factor comprobando que, considerando todas las porciones en conjunto, había diferencias estadísticamente significativas entre las tres especies de moluscos $(\mathrm{p}=$ 0,013 ); pero al aplicar el mismo procedimiento considerando por separado las porciones, sólo hubo diferencias estadísticamente significativas para el líquido de cobertura $(\mathrm{p}=0,037)$, si bien éstas pueden ser debidas más a la naturaleza del líquido que a la influencia de la especie.

Al estudiar si existían diferencias dentro de cada especie (ANOVA) y discriminar sobre qué porciones se mostraban, resultaron sólo significativas para los

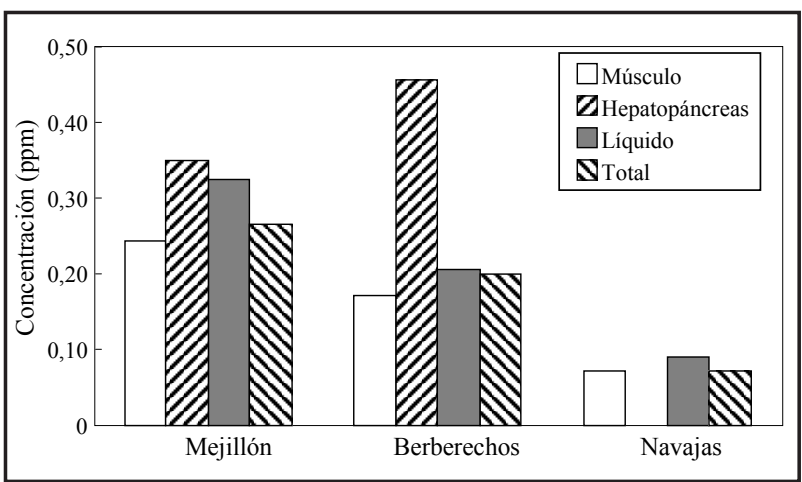

Figura 1. Concentración media de mercurio expresada en ppm $(\mathrm{mg} / \mathrm{kg})$ en peso fresco.

Figure 1. Average concentration of $\mathrm{Hg}$ expressed as $\mathrm{ppm}(\mathrm{mg} / \mathrm{kg})$ in fresh weight.

berberechos $(p=0,035)$ y concretamente entre hepatopáncreas y líquido de cobertura $(\mathrm{p}=0,023)$, según la aplicación del Test t para muestras independientes.

Finalmente, no se observaron diferencias significativas $(p>0,05)$ entre las variadas preparaciones de mejillones objeto de estudio, si bien la preparación «al natural» presentó siempre una concentración más baja de mercurio que el resto de las preparaciones.

Globalmente, los niveles de mercurio obtenidos en este estudio para molusco completo $(0,10-2,14 \mathrm{mg} / \mathrm{kg}$ p.s. $)$ son más altos que los encontrados $(0,04-0,84 \mathrm{mg} / \mathrm{kg}$ p.s.) por Otchere et al. (2003) que analizaron moluscos bivalvos (mejillones y ostras) en diferentes lagos de Ghana. Sin embargo, los niveles encontrados por Beiras et al. (2002) y Besada et al. (2002) son muy similares a los obtenidos en este trabajo. Estos autores estudiaron la contaminación de las costas de Galicia analizando, entre otros, mejillones silvestres, oscilando las concentraciones de mercurio entre 0,101 y $1,109 \mathrm{mg} / \mathrm{kg}$ p.f.

Por otra parte, las concentraciones de mercurio en este estudio son inferiores a las obtenidas por Berzas Nevado et al. (2003) para mejillones $(1,57 \mathrm{mg} / \mathrm{kg}$ p.s.) aunque estos altos niveles se atribuyen a la explotación de una mina de cinabrio próxima al río Valdeazogues (Almadén). También son inferiores a los encontrados para mejillones (1,8-2,6 mg/kg p.s.) por Balagot et al. (2003) en muestras recogidas en Filipinas durante un año y a los obtenidos por Palmer y Presley (1996) en mejillones (0,11$2,52 \mathrm{mg} / \mathrm{kg}$ p.s.) en estuarios contaminados.

En estudios similares al presente trabajo, realizados en conservas de moluscos, Gutiérrez et al. (2004, 2006) obtuvieron contenidos de mercurio muy inferiores a los de este estudio, si bien contrasta que relativamente mientras en los resultados aquí presentados destacan los niveles en el mejillón, para ellos el berberecho fue la especie más acumuladora. Sin embargo, coincide en que la navaja fue la especie menos contaminada. Al igual que en estos trabajos referidos, se observó en otros sobre metales pesados en moluscos en conserva y en fresco (Hernández- 


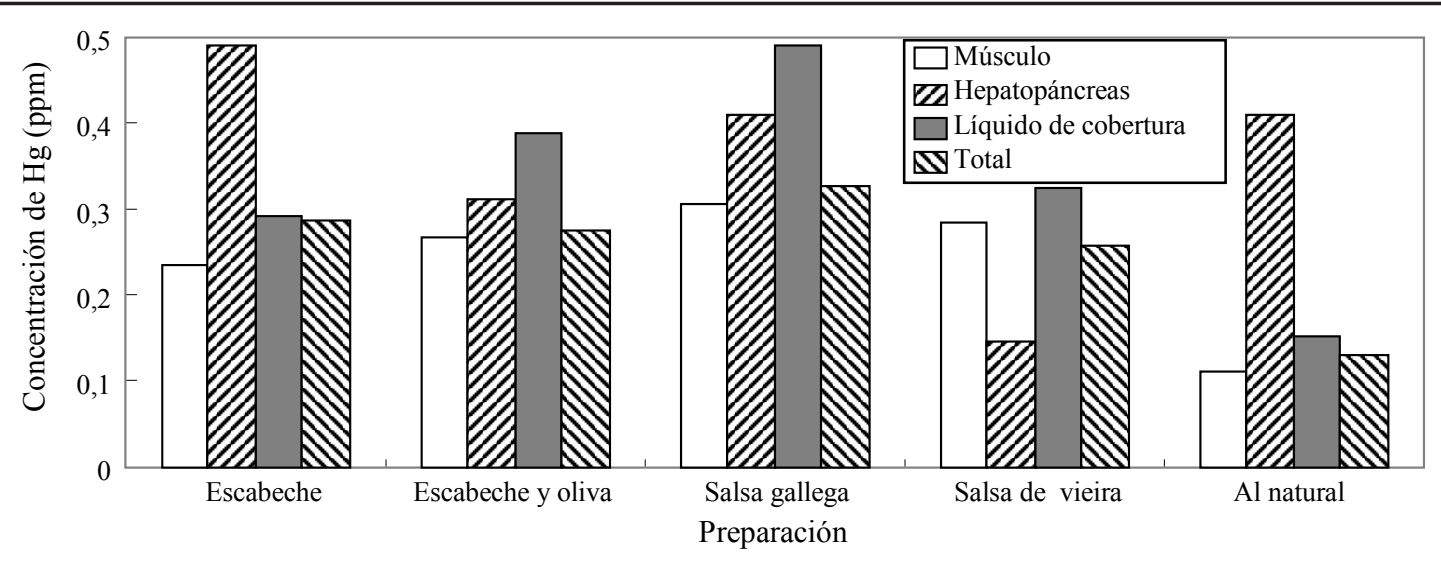

Figura 2. Concentración media de mercurio expresada en ppm $(\mathrm{mg} / \mathrm{kg})$ en peso fresco de mejillones comercializados con distintas preparaciones.

Figure 2. Average concentration of $\mathrm{Hg}$ expressed in $\mathrm{ppm}(\mathrm{mg} / \mathrm{kg})$ in fresh weight of mussels commercialized in several preparations.

Moreno et al., 2004, 2005; Hernández et al., 2006), que no se sobrepasaron los límites exigidos por la legislación vigente, para un consumo habitual. Sin embargo, en un trabajo llevado a cabo por Fang et al. (2001) para 14 especies de moluscos comercializados en fresco en Hong Kong y Guangdong, y para 8 metales, se mostró que el Cd y el $\mathrm{Cr}$ superaron en 5 y 7 especies, respectivamente, los niveles recomendados por la $\mathrm{FAO} / \mathrm{OMS}$.

Considerando la importancia de estos moluscos en la alimentación, el consumo de una lata de conservas de $120 \mathrm{ml}$ de capacidad, con un peso escurrido entre 61-67 g (dependiente de la especie) supone la ingesta media de mercurio de $17,8 \mu \mathrm{g}$ por lata de mejillones; $12,6 \mu \mathrm{g}$ por lata de berberecho y $4,5 \mu \mathrm{g}$ por lata de navajas.

La ingesta semanal provisional tolerable (PTWI) recomendada de mercurio total por la FAO/OMS es de 5 $\mu \mathrm{g} / \mathrm{kg}$ pc, por tanto el límite semanal quedaría fijado en $350 \mu \mathrm{g} /$ persona (suponiendo un peso medio de $70 \mathrm{~kg}$ ). Esta cantidad se alcanzaría consumiendo 20 latas de mejillones, 28 de berberechos o 78 de navajas, consumos que parecen poco probables en una dieta equilibrada, si bien hay que tener en cuenta que el mercurio total ingerido puede tomarse también, a través de otros alimentos. Además, es importante considerar que, aproximadamente el $30 \%$ del mercurio total presente en moluscos se encuentra en forma de metilmercurio (Liang et al., 2003) y el comité de expertos de la FAO/OMS establecieron la ingesta semanal provisional tolerable para el metilmercurio en $1,6 \mu \mathrm{g} / \mathrm{kg}$ p.c., por lo que para una persona adulta de $70 \mathrm{~kg}$ de peso, el límite semanal se situaría en $112 \mu \mathrm{g} /$ persona, lo que supondría consumir al menos 21 latas de mejillones, $30 \mathrm{de}$ berberechos o 83 de navajas, consumos muy poco probables. De acuerdo al Panel de Consumo (2005), en Galicia se ingiere una media de 0,26 kg de mejillones, berberechos y navajas en conserva por persona y año, esto supone aproximadamente $0,005 \mathrm{~kg} / \mathrm{semana}$ para estos moluscos, valor bastante alejado del límite recomendado (112 $\mu \mathrm{g} /$ persona/semana).

\section{CONCLUSIONES}

El hepatopáncreas fue la porción anatómica que presentó las concentraciones más elevadas de mercurio en las especies estudiadas. Las navajas mostraron los índices más bajos de contaminación, ofreciendo además, mayor seguridad alimentaria la extracción del hepatopáncreas en los preparados comerciales. Teniendo en cuenta la participación de estos moluscos en conserva en la dieta gallega, puede considerarse que el aporte tanto de mercurio como de metilmercurio no supone riesgo de tipo toxicológico.

\section{AGRADECIMIENTOS}

A la Xunta de Galicia que subvencionó el Proyecto PGIDIT02TAL26101PR.

\section{BIBLIOGRAFÍA}

Alonso, J.; Salgado, M. J.; García, M. A.; Melgar, M. J. 2000. Accumulation of mercury in edible macrofungi: influence of some factors. Archives of Environmental Contamination and Toxicology 38, 158-162.

Balagot, A. H.; Villaflor, A. C.; Sánchez, F. C.; Develles, E. N. 2003. $\mathrm{Pb}, \mathrm{Cd}$ and $\mathrm{Hg}$ contents of bivalves collected during the different seasons of the year. Food Research Program 54, 256.

Beiras, R.; Fernández, N.; González, J. J.; Besada, V.; Schultze, F. 2002. Mercury concentrations in seawater, sediments and wild mussels from the coast of Galicia (NW Spain). Marine Pollution Bulletin 44, 340-349. 
Berzas Nevado, J. J.; García Bermejo, L. F.; Rodríguez Martín-Doimeadios, R. C. 2003. Distribution of mercury in the aquatic environment at Almadén, Spain. Environmental Pollution 122, 261-271.

Besada, V.; Fumega, J.; Vaamonde, A. 2002. Temporal trenes of $\mathrm{Cd}, \mathrm{Cu}, \mathrm{Hg}, \mathrm{Pb}$ and $\mathrm{Zn}$ in mussel (Mytilus galloprovincialis) from the Spanish North-Atlantic coast 1991-1999. The Science of the Total Environmental 288, 239-253.

BOE, 1993. Real Decreto 308/1993 de 25 de febrero, por el que se aprueba la reglamentación técnico-sanitaria que fija las normas aplicables a la comercialización de moluscos bivalvos vivos (BOE de 30/3/1993).

BOE, 2003. Real Decreto 256/2003, de 28 de febrero, por el que se fijan los métodos de toma de muestras y de análisis para el control oficial del contenido máximo de plomo, cadmio, mercurio y 3-monocloropropano1,2-diol en los productos alimenticios (BOE de 1/3/ 2003).

DOCE, 1990. Decisión de la Comisión, de 26 de septiembre de 1990, por la que se establecen los métodos de referencia para la investigación de residuos de metales pesados y de arsénico (DOUE de 18/10/ 1990).

Domouhtsidou, G. P.; Dimitriadis, V. K. 2000. Ultrastructural localization of heavy metals $(\mathrm{Hg}, \mathrm{Ag}, \mathrm{Pb}$ and $\mathrm{Cu})$ in gills and digestive gland of mussels, Mytilus galloprovincialis (L.). Archives of Environmental Contamination and Toxicology 38, 472-478.

DOUE, 2006. Reglamento (CE) 1881/2006 de la Comisión, de 19 de diciembre de 2006 por el que se fija el contenido máximo de determinados contaminantes en los productos alimenticios (DOUE de 20/12/ 2006).

Fang, Z.; Cheung, R. Y. H.; Wong, M. H. 2001. Heavy metal concentrations in edible bivalves and gastropods available in major markets of the Pearl River Delta. Journal of Environmental Sciences 13, 210-217.

García-Vázquez, M. 2005. Evaluación del contenido de mercurio y su repercusión toxicológica en conservas de zamburiñas y almejas. Proyecto Fin de Carrera. Área de Toxicología. Facultad de Ciencias de Lugo. Universidad de Santiago de Compostela.

Gobert, S.; Daemers-Lambert, C.; Bouquegneau, J.M. 1992. Etat physiologique et contamination en metaux lourds des moules Mytilus edulis sur la Côte Belge. Bulletin de la Société Royale des Sciences de Liège 61, 177-194.

Gutiérrez, A. J.; Lozano, G.; Hardisson, A.; Rubio, C.; González, T. 2004. Content of toxic and essential metals in canned mussels commonly consumed in Tenerife, Canary Islands, Spain. Journal of Food Protection 67, 1526-1532.

Gutiérrez, A. J.; Lozano, G.; González, T.; Ignacio Reguera, J.; Hardisson, A. 2006. Mercury content in tinned molluscs (mussel, cockle, variegated scallop, and razor shell) normally consumed in Spain, 2005. Journal of Food Protection 69, 2237-2240.

Hernández-Moreno, D.; García Fernández, M. A.; Alonso Díaz, J.; Melgar Riol, M. J.; Pérez López, M. 2004. Concentraciones de metales pesados en conservas de almeja, berberecho y navaja comercializadas en España. Ciencia y Tecnología Alimentaria 4, 197205.

Hernández-Moreno, D.; García Fernández, M. A.; Alonso Díaz, J.; Pérez López, M.; Melgar Riol, M. J. 2005. Estudio comparativo sobre los niveles de metales pesados en almejas (Mulinia spp.) en conserva y en fresco comercializados en Galicia. Ciencia y Tecnología Alimentaria 5, 324-329.

Hernández, D.; García, M. A.; Pérez-López, M.; Melgar Riol, M. J. 2006. Niveles de metales pesados (zinc, cobre, cadmio y plomo) en navajas (Ensis ensis L.) en fresco en conserva y en fresco. Alimentaria 376, 94-96

Klaassen, C. D. 2001. Casarett and Doull's Toxicology: the basic science of poisons. Ed. McGraw-Hill. $6^{\text {th }}$ ed. New York.

Liang, L; Shi, J.; He, B.; Jiang, G.; Yuan, C. 2003. Investigation of methylmercury and total mercury contamination in mollusc samples collected from coastal sites along the Chinese Bohai Sea. Journal of Agrigultural and Food Chemistry 51, 7373-7378.

Mas, A.; Azcue, J. M. 1993. Metales en sistemas biológicos. Promociones y Publicaciones Universitarias, S.A., Barcelona.

Otchere, F. A.; Joiris, C. R.; Holsbeek, L. 2003. Mercury in the bivalves Anadara (Senilia) senilis, Perna perna and Crassostrea tulipa from Ghana. The Science of the Total Environmental 304, 369-375.

Palmer, S. J.; Presley, B. J. 1996. Mercury concentrations in benthic organisms from a contaminated estury. Marine Environmental Research 41, 225-239.

Prego, R. 1995. Aspectos generales de la circulación dinámica de sales nutrientes y contaminación en las aguas de las Rías Gallegas. Instituto de Investigaciones Marinas (C.S.I.C.), Vigo.

Puerta, 1995. La depuración de los moluscos bivalvos. Fundación Caixa Galicia, Aula del Mar. Vigo.

Riisgård, H.U.; Kiørboe, T.; Møhlenberg, F.; Drabæk, I.; Madsen, P. 1985. Accumulation, elimination and speciation of mercury in the bivalves Mytilus edulis and Macoma Baltica. Marine Biology 86, 52-55.

Vázquez, J. L. 2002. Consecuencias de la marea roja en el ser humano. Gaceta CUC 2(2), 11.

Wang, W. X. 2001. Comparison of metal uptake and absorption efficiency in marine bivalves. Environment Toxicology and Chemistry 20(6), 1367-1373. 